\title{
Optimization of force-limiting seismic devices connecting structural subsystems
}

\author{
Alessandro Scodeggio ${ }^{\mathrm{a}}$, Giuseppe Quaranta ${ }^{\mathrm{b}, 1}$, Giuseppe C. Marano ${ }^{\mathrm{c}}$, \\ Giorgio Monti ${ }^{\mathrm{d}}$, Robert B. Fleischman ${ }^{\mathrm{e}}$ \\ ${ }^{a}$ Department of Structural Engineering, National Technical University of Athens, 5 Iroon \\ Polytechniou, 15773 Zografou, Athens, Greece \\ ${ }^{b}$ Department of Structural and Geotechnical Engineering, Sapienza University of Rome, \\ via Eudossiana 18, 00184 Rome, Italy \\ ${ }^{c}$ Department of Civil Engineering and Architecture, Technical University of Bari, via \\ Orabona 4, 70125 Bari, Italy \\ ${ }^{d}$ Department of Structural and Geotechnical Engineering, Sapienza University of Rome, \\ via Gramsci 53, 00197 Rome, Italy \\ ${ }^{e}$ Department of Civil Engineering and Engineering Mechanics, School of Engineering, \\ University of Arizona, 1209 E. 2nd St., Tucson, AZ 85721, United States
}

\begin{abstract}
This paper is focused on the optimum design of an original force-limiting floor anchorage system for the seismic protection of reinforced concrete (RC) dual wall-frame buildings. This protection strategy is based on the interposition of elasto-plastic links between two structural subsystems, namely the lateral force resisting system (LFRS) and the gravity load resisting system (GLRS). The most efficient configuration accounting for the optimal position and mechanical characteristics of the nonlinear devices is obtained numerically by means of a modified constrained differential evolution algorithm. A 12-storey prototype RC dual wall-frame building is considered to demonstrate the effectiveness of the seismic protection strategy.
\end{abstract}

Keywords: differential evolution; force-limiting floor anchorage system; optimization; seismic protection.

Email address: giuseppe.quaranta@uniroma1.it (Giuseppe Quaranta)

${ }^{1}$ Corresponding author. Tel.: +39 (06) 44585294. 


\section{Introduction}

The protection of structures in earthquake-prone regions may require the installation of devices for the mitigation of the demand induced by seismic loads, such as active control systems [1], single or multiple passive tuned mass dampers [2-4] or base isolators [5, 6]. Throughout the available technologies for seismic protection, passive devices are especially widespread because of their robustness, low manufacturing cost (compared to the cost of the whole building) and easy maintenance. The use of additional mechanical and/or hydro-mechanical devices often needs a design strategy capable of defining the optimal configuration that would ensure an adequate reduction of the seismic demand.

Among the existing strategies for seismic protection, the use of devices for connecting adjacent structures has been investigated in the past decades. In this regard, the use of viscous dampers between two neighboring floors is quite common [7], typically together with a linear spring within a Voigtor Maxwell-type configuration [8,9]. Other studies consider nonlinear models, such as a friction model [10] or the Bouc-Wen model [11, 12]. For what concerns the behavior of the coupled buildings, basically all studies assume shear-type linear elastic and viscously damped systems having one or multiple degrees-of-freedom [8-12]. Existing literature encompasses analytical or semi-analytical approaches as well as numerical procedures based on soft computing techniques. For instance, Zhu and $\mathrm{Xu}$ [9] presented closed formulations to estimate the optimal parameters of Maxwell model-defined fluid dampers connecting adjacent structures under white-noise ground excitation. The stochastic equivalent linearization technique was exploited in [13] and an energy performance index is determined by calculating the stochastic response of two interconnected structural systems. In this case, the optimal device is the one that corresponds to the maximum value of the computed performance index. Differently from rigorous analytical approaches, empirical design procedures based on the dynamic characteristics of the structure have the merit of allowing the design of seismic protection strategies by employing a lower computational effort, see for instance [14] for an application to the optimal placement of controllers. On the other hand, multi-objective optimization problems were proposed in [15, 16], and a genetic algorithm was considered to find the Pareto optimal solutions. Both studies considered linear elastic shear-type protected systems. Particularly, Uz and Hadi [16] studied the simultaneous minimization of two objective functions, i.e. 
the maximum relative displacement between two successive floors and the total number of semi-active magnetorheological dampers. The dampers were modeled using the Bouc-Wen model and the motion equation was linearized in a stochastic sense. Pareto optimal solutions were calculated via genetic algorithm for two different earthquake records.

The passive seismic protection strategy investigated in this paper is based on the use of energy dissipation devices (nonlinear connectors) to be placed between the lateral force resisting system (LFRS) and the gravity load resisting system (GLRS). This type of protection system is especially intended for mid-rise structures, such as conventional residential buildings, hospitals, schools, laboratories, institutions and commercial buildings. The devices are positioned with their vertical axis lying on the horizontal plane of the corresponding slab, to which they are connected at one edge. On the opposite side, they are fixed to the LFRS. Therefore, the force developed by the device acts on the same plane of the slab to which it is linked. The connection between GLRS and LFRS is initially elastic. Once a predefined horizontal force level is achieved - which could be either the static friction force for friction devices or the yield force for other elasto-plastic devices - the primary transfer mechanism is activated by experiencing a nonlinear behavior, thus starting to dissipate energy through the relative motion between GLRS (e.g., floors system and gravity columns) and LFRS (e.g., cores, shear walls, braced or moment resisting frames). This relative displacement of the floor is controlled and limited by the displacement capacity of the device. After an earthquake, only the connectors that suffered high deformations have to be replaced, without damages to the other structural members of the GLRS (which have to behave elastically under the design displacement of the seismic protection system). In this way, the permanent deformations of the building are removed and the system is brought to its initial configuration. The presence of these seismic devices uncouples the response of shear wall and floors system, and thus it enables the design of the GLRS by mainly referring to the gravity loads. Since the seismic forces carried by buildings originate mostly from the inertia of the floor system, a reduction of the accelerations transmitted between LFRS and GLRS leads to lower demands for the structural elements, thus mitigating structural and non-structural damages.

Indeed, the working principle of such protection system is similar to that exploited when coupling adjacent buildings, i.e. it makes use of the fact that dynamic responses of dissimilar systems are different under the same ground motion. Nonetheless, the seismic protection system here considered is some- 
what different. Most important, in this study two subsystems of one building (namely, LFRS and GLRS) are connected each other, against the typical situation that occurs if two adjacent buildings are coupled, where two systems are connected. The considered seismic protection strategy is best suited for new buildings, even if it represents a feasible choice for the retrofitting of existing RC buildings. Concerning possible issues arising from the installation of the nonlinear connectors, practical problems will be certainly faced when placing these devices within the structural system of an existing RC building, whereas in new buildings the subsystems can be designed so as to best incorporate the connectors. On the other hand, the connection of two adjacent buildings may result quite problematic if they belong to different owners. Hence, eventual disputes are avoided when connecting structural subsystems, because the intervention concerns the same building.

Within this framework, the optimum design of elasto-plastic devices to be placed between GLRS and LFRS is hereafter investigated in order to demonstrate the effectiveness of the seismic protection strategy. Further aspects regarding different mechanical characteristics, improved nonlinear modeling, constructive and technological details of the connectors will be topics of future insights. Differently from the previous studies in similar research areas, this paper considers a realistic structural modeling for both subsystems, whereby the performance under seismic loads is evaluated through nonlinear dynamic analyses. Based on these assumptions, the resulting constrained seismic design optimization problem cannot be solved analytically and, as a consequence, a computer-aided procedure that exploits an advanced differential evolution algorithm has been implemented. The optimization strategy has been developed in MATLAB whereas the OpenSees platform has been used for nonlinear dynamic analysis. Results for a 12-storey prototype reinforced concrete (RC) dual wall-frame building are discussed at the end of the paper for demonstrating the effectiveness of such seismic protection strategy.

\section{Optimum design problem}

The seismic performance of the protected building depends on, both, mechanical properties and vertical distribution of the passive devices connecting LFRS and GLRS. Choosing optimal mechanical characteristics and position for such floor connectors is not a straightforward numerical task, as either favorable or unfavorable results can be achieved. The search for favorable 
links' configurations and properties is hereafter formulated as constrained single-objective optimization problem.

\subsection{Formulation}

The optimum design problem of this seismic protection strategy aims at minimizing a cost-based objective function under the condition that appropriate constraints (depending on the performance of the protected structural system) are satisfied. Therefore, it is mathematically formulated as singleobjective constrained optimization problem:

$$
\begin{aligned}
& \min _{\mathbf{x} \in \mathcal{D}}\{f(\mathbf{x})\} \\
& \text { s.t. } \\
& g_{a}(\mathbf{x}) \leq 0
\end{aligned}
$$

where $f(\mathbf{x})$ is the objective function, $\mathbf{x}$ is the design vector and $g_{a}(\mathbf{x})$ are constraints of the optimization problem (with $a=1, \ldots, N C$ ). The design vector $\mathbf{x}$ is lower bounded by $\mathbf{x}^{l}$ and upper bounded by $\mathbf{x}^{u}$. These bounds define the hyper-rectangle $\mathcal{S}$, that is the total search space of the problem. The best design solution $\mathbf{x}^{*}$ is the global minimum of the objective function within the feasible domain $\mathcal{D} \subset \mathcal{S}$.

\subsection{Design variables}

Floor connectors are modeled as elastic-perfectly plastic springs with no supplemental damping. The vector $\mathbf{x}_{i}=\left\{\begin{array}{lllll}x_{i 1} & \ldots & x_{i j} & \ldots & x_{i n}\end{array}\right\}$ denotes the $i$ th candidate design solution and it includes the stiffness value of each link, which is assumed as a continuous variable. For instance, $x_{i j}$ is the $i$ th candidate stiffness link value at the $j$ th floor. The strength value of each link depends on the selected device, and it can be (linear or nonlinear) function of the stiffness. In doing so, the strength is not considered as a constant value because it depends on the corresponding optimal stiffness value. As the stiffness of the links are the independent design parameters of the optimization problem, the number of dimensions of the search space is restricted to the number of floor levels (whereas it doubles when considering the strength value of each link as an independent design parameter). Although every candidate design solution $\mathbf{x}_{i}$ is defined through $n$ stiffness values, the building may not have all $n$ possible floor connectors installed. The placement of a link is based on the corresponding design variable value 


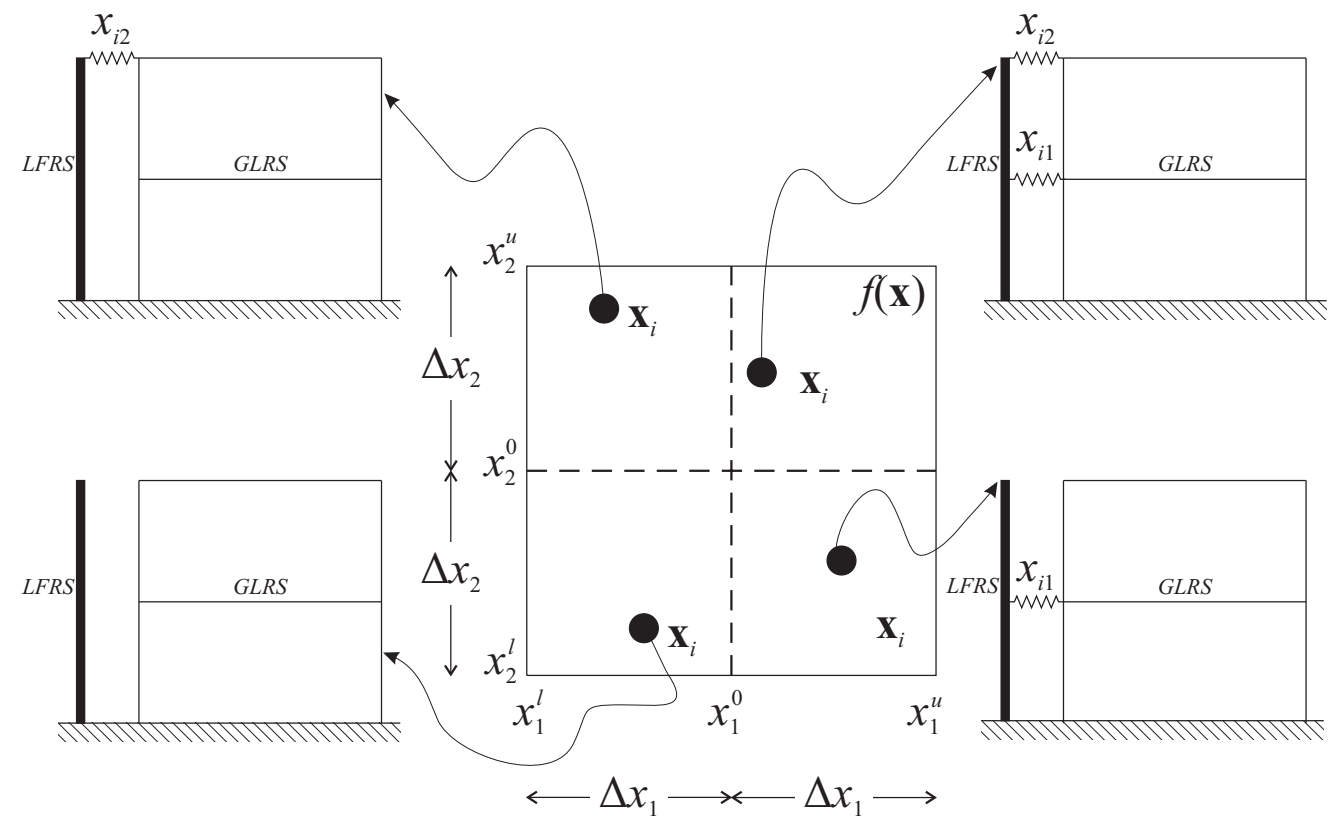

Figure 1: Placement of links between the LFRS and the GLRS: adopted strategy to identify operative floor connectors within a bi-dimensional search space.

by adapting an idea presented in [17] and recently implemented in [18] to look for the best seismic isolators configuration using a genetic algorithm. On assuming $x_{j}^{0}=x_{j}^{l}+\Delta x_{j}=x_{j}^{l}+0.5\left(x_{j}^{u}-x_{j}^{l}\right.$ ) (where $x_{j}^{l}$ and $x_{j}^{u}$ are the $j$ th components of $\mathbf{x}^{l}$ and $\mathbf{x}^{u}$, respectively), the floor connector at the $j$ th floor is operative if $x_{j}^{0}<x_{i j} \leq x_{j}^{u}$ and it does not exist otherwise. Therefore, the vectors $\mathbf{x}^{0}$ and $\mathbf{x}^{u}$ are the physical bounds of the design vector $\mathbf{x}$. On the contrary, the $j$ th floor connector is too flexible if $x_{j}^{l} \leq x_{i j} \leq x_{j}^{0}$, and thus does not provide an effective link between the GLRS and the LFRS. The (fictitious) bound $\mathbf{x}^{l}$ is then defined in such a way that an equal probability of being operative or inexistent is given to any floor connector in the building. The adopted approach is graphically explained in Figure 1 when the objective function $f(\mathbf{x})$ takes real values over a bidimensional search space $\mathcal{S}$ (that is, in two-storey building structures). This strategy has the merit to allow the use of real positive numbers to represent the link stiffness without introducing additional variables in order to codify the links' configuration. 


\subsection{Objective function}

The objective function of the optimization problem is the total cost of the seismic protection system:

$$
f(\mathbf{x})=\sum_{j \in \mathcal{L}} c_{j}(\mathbf{x})
$$

where $\mathcal{L}=\left\{j: x_{j}^{0}<x_{j} \leq x_{j}^{u}\right\}$ is the set collecting operative floor connectors and $c_{j}(\mathbf{x})$ is the cost associated with the implementation of the $j$ th link. The cost $c_{j}(\mathbf{x})$ can include, both, manufacturing costs (which depend on the selected device) and installation costs. However, it is not necessary to use real costs in order to apply Eq. (2). On assuming $c_{j}(\mathbf{x})$ a linear function of the link stiffness $x_{j}$, the objective function in Eq. (2) reduces to:

$$
f(\mathbf{x})=\sum_{j \in \mathcal{L}} x_{j}
$$

which can be used without a priori information about the manufacturing cost of the device to be installed. The cost function in Eq. (3), however, can be easily reformulated once the class of devices to be installed has been selected.

\subsection{Constraints}

The optimal mechanical properties and positions of the floor connectors are selected to minimize the objective function while satisfying appropriate limit states. Problem constraints concern with the structural performance and the operating conditions of the floor connectors.

Inter-storey drifts. The protection system is designed to keep the inter-storey drift values below a given threshold. The maximum allowable drift value depends on the desired structural performance. It, in turn, depends on the considered building and the acceptable damage severity. Therefore, the following drift-based constraint is assumed:

$$
\max _{\tau}\left\{\delta_{j}\left(\mathbf{x} \mid a_{g}(\tau)\right)\right\} \leq \delta_{\max }
$$

where $\delta_{j}\left(\mathbf{x} \mid a_{g}(\tau)\right)$ is the inter-storey drift of the $j$ th storey under the ground acceleration $a_{g}(\tau)$ for an assigned design vector $\mathbf{x}$ ( $\tau$ is the time parameter) whereas $\delta_{\max }$ is the maximum allowable inter-storey drift value. Imposing 
a constraint to the inter-storey drift demand for both GLRS and LFRS is of primary importance for the preservation of the structural integrity and the prevention of the damage induced by earthquakes, especially on vertical structural members and walls. An inter-storey drift limitation is also important to minimize damage and prevent fall out of non-structural elements or building equipments that are sensitive to relative displacements between storeys.

Absolute accelerations. The (absolute) floor acceleration is often considered as a performance index related to non-structural damages and should be limited to a certain value that depends on the type of building. The accelerationbased constraint of the optimization problem is the following:

$$
\max _{\tau}\left\{a_{j}\left(\mathbf{x} \mid a_{g}(\tau)\right)\right\} \leq a_{\max }
$$

where $a_{j}\left(\mathbf{x} \mid a_{g}(\tau)\right)$ is the acceleration at the $j$ th storey under the ground acceleration $a_{g}(\tau)$ for an assigned design vector $\mathbf{x}$ whereas $a_{\text {max }}$ is the maximum acceleration value. Considering the fact that this type of protection system is also intended for important buildings (e.g., hospitals, schools, laboratories, institutions and commercial buildings characterized by the presence of expensive content and equipments), it is certainly required to limit the seismic demand in terms of floor accelerations. For this reason, the absolute acceleration of the GLRS (i.e., the acceleration at the floors level) must be lower than a predefined threshold in order to minimize non-structural damages. For the structural configuration here investigated, the LFRS is represented by a shear wall that is free of sensitive furniture and equipments. Therefore, floor accelerations of the LFRS are not monitored.

Sliding. Any isolation device is designed and manufactured to operate within certain deformation limits. Therefore, the relative displacement between the LFRS and the GLRS should be limited within a prescribed range. Hence, it has to be ensured that the displacement demand of the optimized system remains within the limits specified by the manufacturer, thus preventing the link failure. As a consequence, the following constraint is also imposed:

$$
\max _{\tau}\left\{d_{j}\left(\mathbf{x} \mid a_{g}(\tau)\right)\right\} \leq d_{\max }
$$

where $d_{j}\left(\mathbf{x} \mid a_{g}(\tau)\right)$ is the relative displacement between the LFRS and the floor system (GLRS) at the $j$ th storey under the ground acceleration $a_{g}(\tau)$ 
for an assigned design vector $\mathbf{x}$ whereas $d_{\max }$ is the corresponding maximum allowable value.

\section{Optimization procedure}

Structural performance and operating conditions of the floor connectors will be evaluated through nonlinear simulations using real earthquake records and an equivalent bidimensional finite element model representing the structural system layout. Under these assumptions and because of the nonlinear constraints, no analytical approaches can be used and thus an efficient numerical technique for the optimization of this seismic protection strategy is required. The development of optimization methods able to handle the nonlinear behavior of RC structures is a challenging computational task. A remarkable result in this field has been obtained in [19], in which the program D.O.T. was used for performing the structural optimization of a RC portal frame according to the NHERP Guidelines whereas the software DRAIN-2DX was used for carrying out the pushover analysis.

Within the presented work, the nonlinear behavior of the coupled structural subsystems is evaluated through nonlinear dynamic analyses whereas the optimization is performed by means of the $(\mu+\lambda)$-constrained differential evolution algorithm [20], see also [21]. The differential evolution algorithm proposed in [20] has been herein modified by implementing an eigenvectorbased crossover operator. The adopted numerical optimization strategy is now presented by focusing on the most important implementation issues.

\subsection{Basic operations in differential evolution}

Among the available nature-inspired paradigm to solve optimization problems, the DE has proved to be very effective, resulting among the most popular in structural engineering applications, e.g. [22]. Its search mechanism is based on the manipulation of $N P$ candidate solutions (or individuals) $\mathbf{x}_{i}$ (with $i=1, \ldots, N P$ ) at each iteration $t$. The first operator is the socalled mutation which performs a perturbation on the candidate solution by using the differences between couples of selected individuals. The $(\mu+\lambda)$ constrained differential evolution is based on the following mutation operators:

$$
\begin{gathered}
\mathbf{v}_{i}=\mathbf{x}_{r 1}+F\left(\mathbf{x}_{r 2}-\mathbf{x}_{r 3}\right) \\
\mathbf{v}_{i}=\mathbf{x}_{r 1}+F\left(\mathbf{x}_{r 2}-\mathbf{x}_{r 3}\right)+F\left(\mathbf{x}_{r 4}-\mathbf{x}_{r 5}\right)
\end{gathered}
$$




$$
\begin{gathered}
\mathbf{v}_{i}=\mathbf{x}_{i}+F\left(\mathbf{x}_{r 1}-\mathbf{x}_{i}\right)+F\left(\mathbf{x}_{r 2}-\mathbf{x}_{r 3}\right) \\
\mathbf{v}_{i}=\mathbf{x}_{i}+F\left(\mathbf{x}_{\text {best }}-\mathbf{x}_{i}\right)+F\left(\mathbf{x}_{r 1}-\mathbf{x}_{r 2}\right)
\end{gathered}
$$

which are dubbed "rand/1", "rand/2", "current-to-rand/1" and "currentto-best/1", respectively. In Eq. (7), $\mathbf{v}_{i}=\left\{\begin{array}{lllll}v_{i 1} & \ldots & v_{i j} & \ldots & v_{i n}\end{array}\right\}$ is the mutant vector, $r 1, \ldots, r 5$ are integers randomly selected from $\{1, \ldots, N P\}$ such that $r 1 \neq r 2 \neq r 3 \neq r 4 \neq r 5, F$ is the mutation (or scaling) factor and $\mathbf{x}_{\text {best }}$ is the best individual in the population. The feasibility of $\mathbf{v}_{i}$ is ensured by adopting a projection scheme. This operation modifies each out-of-bound component of $\mathbf{v}_{i}$ using the following rule:

$$
v_{i j}=\left\{\begin{array}{ll}
2 x_{j}^{l}-v_{i j} & \text { if } v_{i j}<x_{j}^{l} \\
2 x_{j}^{u}-v_{i j} & \text { if } v_{i j}>x_{j}^{u} \\
v_{i j} & \text { otherwise }
\end{array} .\right.
$$

The binomial crossover operator is defined in such a way that $\forall j$ :

$$
u_{i j}= \begin{cases}v_{i j} & \text { if } \text { rand } \leq C R \text { or } j=j_{\text {rand }} \\ x_{i j} & \text { otherwise }\end{cases}
$$

where rand is a uniformly distributed random number between 0 and 1 , $j_{\text {rand }}$ is a randomly selected integer from 1 to $n, C R$ is the crossover rate. The eigenvector-based operator is built on the binomial crossover and is implemented as follows [23]:

$$
\mathbf{u}_{i}= \begin{cases}\mathbf{Q}^{* T} \text { xover }\left(\mathbf{Q} \mathbf{x}_{i}, \mathbf{Q} \mathbf{v}_{i}\right) & \text { if rand } \leq P \\ \text { xover }\left(\mathbf{x}_{i}, \mathbf{v}_{i}\right) & \text { otherwise }\end{cases}
$$

where $\operatorname{xover}(\cdot, \cdot)$ is the binomial crossover, $P$ is the eigenvector ratio, $\mathbf{Q}^{* T}$ is the conjugate transpose of the eigenvector basis $\mathrm{Q}$ (with $\mathrm{C}=\mathrm{Q} \Lambda \mathrm{Q}^{-1}$, being C the covariance matrix over the current population).

The iterative strategy runs until $t \leq T$, where $T$ is the maximum number of iterations.

\subsection{Improved $(\mu+\lambda)$-constrained differential evolution}

The pseudo-code of the $(\mu+\lambda)$-constrained differential evolution proposed in [20] is described in Algorithm 1, together with the external links to the OpenSees platform for nonlinear dynamic analyses. Key components of the optimization procedure are: 
- an improved differential evolution (IDE) algorithm based on the simultaneous implementation of several mutation operators, and

- the archiving-based adaptive trade-off model (ArATM) which selects a proper constraint-handling mechanism for each situation.

\subsection{Degree of constraint violation}

The degree of constraint violation of the candidate solution $\mathbf{x}_{i}$ is computed $\forall a$ :

$$
G_{a}\left(\mathbf{x}_{i}\right)=\max \left\{0, g_{a}\left(\mathbf{x}_{i}\right)\right\} .
$$

Subsequently, the difference $\Delta$ is calculated using Eq. (12):

$$
\Delta=\max _{a}\left\{\max _{i}\left\{G_{a}\left(\mathbf{x}_{i}\right)\right\}\right\}-\min _{a}\left\{\max _{i}\left\{G_{a}\left(\mathbf{x}_{i}\right)\right\}\right\}
$$

and the following flag variable criterion is introduced:

$$
\text { criterion }= \begin{cases}1 & \text { if } \Delta \leq \eta \\ 2 & \text { otherwise }\end{cases}
$$

where $\eta$ is a threshold value, i.e. $\Delta \leq \eta$ implies that the difference among the violations is not too large. If criterion is equal to 1 , then $G\left(\mathbf{x}_{i}\right)$ is calculated by summing the degrees of constraint violation $G_{a}\left(\mathbf{x}_{i}\right)$ in Eq. (11), that is:

$$
G\left(\mathbf{x}_{i}\right)=\sum_{a=1}^{N C} G_{a}\left(\mathbf{x}_{i}\right) .
$$

On the other hand, if criterion is equal to 2 , then $G\left(\mathbf{x}_{i}\right)$ is defined as follows:

$$
G\left(\mathbf{x}_{i}\right)=\frac{\sum_{a=1}^{N C} \bar{G}_{a}\left(\mathbf{x}_{i}\right)}{N C}
$$

where $\bar{G}_{a}\left(\mathbf{x}_{i}\right)$ is:

$$
\bar{G}_{a}\left(\mathbf{x}_{i}\right)=\frac{G_{a}\left(\mathbf{x}_{i}\right)}{\max _{i}\left\{G_{a}\left(\mathbf{x}_{i}\right)\right\}} .
$$

\subsection{Improved differential evolution}

The pseudo-code of the IDE algorithm is given in Algorithm 2. Its output is the offspring population $\mathcal{Q}_{t}$ (consisting of $\lambda$ individuals) obtained from the 


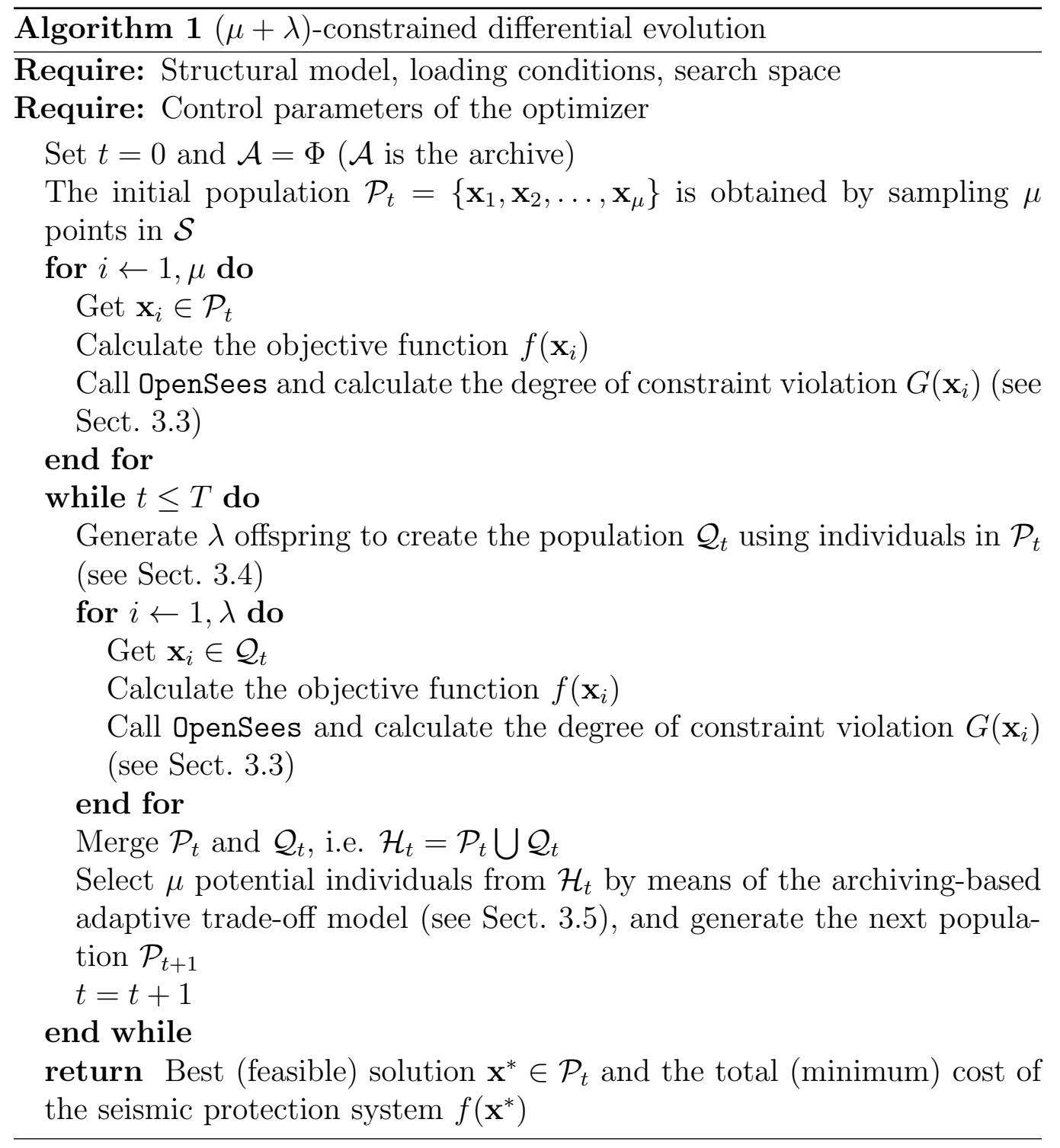


parent population $\mathcal{P}_{t}$ (whose number of individuals is $\mu$ ) by implementing several mutation operators simultaneously.

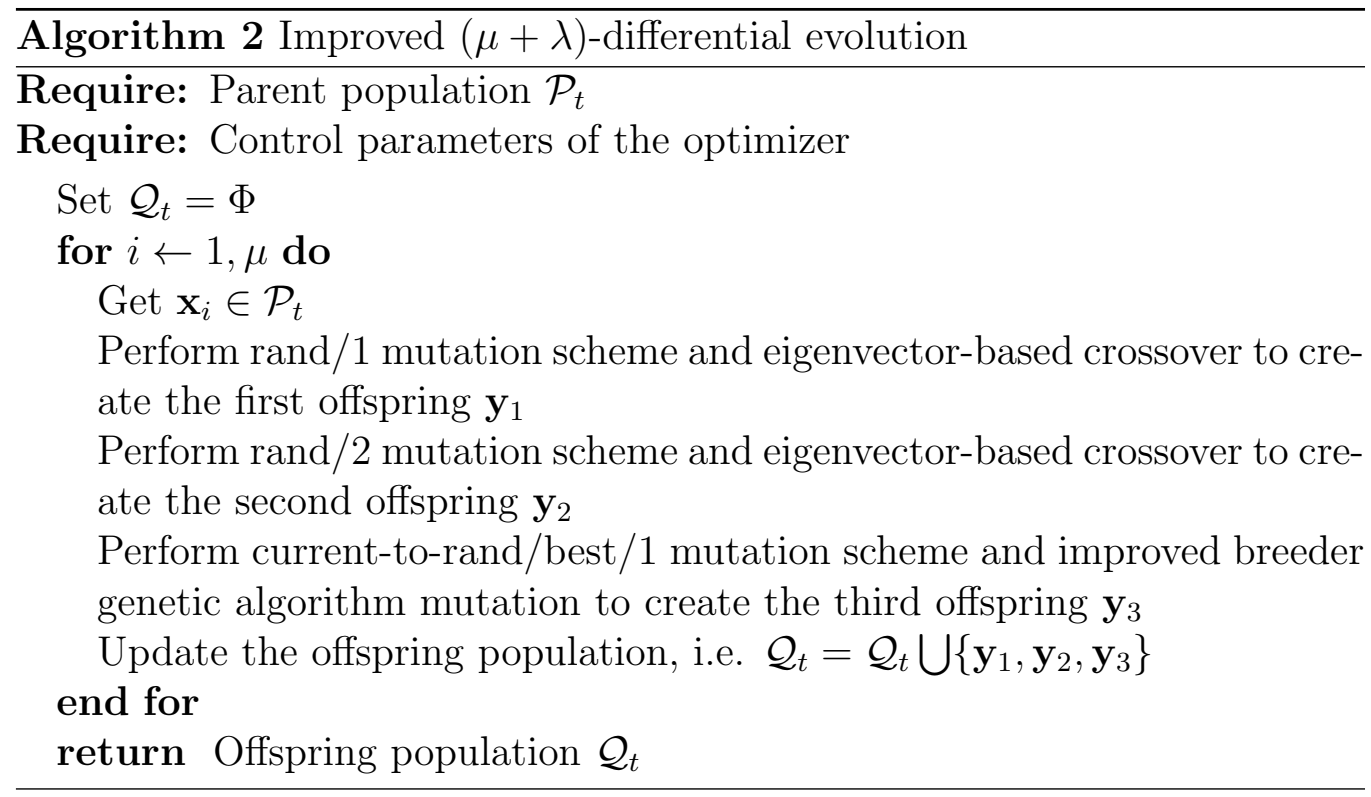

In Algorithm 2, the "current-to-rand/best/1" mutation adopts an iterationdependent criterion to switch from the "current-to-rand/1" strategy in Eq. (7c) to the "current-to-best/1" strategy in Eq. (7d). It works as follows:

$$
\mathbf{v}_{i}= \begin{cases}\mathbf{x}_{i}+F\left(\mathbf{x}_{r 1}-\mathbf{x}_{i}\right)+F\left(\mathbf{x}_{r 2}-\mathbf{x}_{r 3}\right) & \text { if } t \leq \kappa T \\ \mathbf{x}_{i}+F\left(\mathbf{x}_{\text {best }}-\mathbf{x}_{i}\right)+F\left(\mathbf{x}_{r 1}-\mathbf{x}_{r 2}\right) & \text { otherwise }\end{cases}
$$

where $\kappa T$ is the threshold iteration number $(\kappa$ is a positive control parameter less than 1). The third offspring $\mathbf{y}_{3}$ is finally obtained by applying an improved breeder genetic algorithm mutation to $\mathbf{v}_{i}$ with probability $P M$ :

$$
y_{3 j}=\left\{\begin{array}{ll}
v_{i j} \pm \operatorname{rang}_{i} \sum_{s=0}^{15} \alpha_{s} 2^{-s} & \text { if } \text { rand }<\frac{1}{n} \\
v_{i j} & \text { otherwise }
\end{array} .\right.
$$

In Eq. (18), rand is a freshly generated uniform random number between 0 and $1, \operatorname{rang}_{i}=\left(x_{i}^{u}-x_{i}^{l}\right)(1-t / T)^{6}$ is the mutation range, the sign + or - is chosen with probability 0.5 , and $\alpha_{s} \in\{0,1\}$ is a random number generated with the probability $p_{r}\left(\alpha_{s}=1\right)=\frac{1}{16}$. Moreover, if the condition $t \leq \kappa T$ holds, then Eq. (18) is not performed. This implies that $\mathbf{y}_{3}=\mathbf{v}_{i}$ as it results 
from the "current-to-rand/1" scheme in Eq. (17).

\subsection{Archiving-based adaptive tradeoff model}

The combined population $\mathcal{H}_{t}=\mathcal{P}_{t} \cup \mathcal{Q}_{t}$ can include feasible individuals only, infeasible candidate solutions only, both infeasible and feasible individuals. As the formulated optimization problem involves several constraints, the probability of handling a large number of infeasible solutions is very high (especially at the beginning, where it is possible that no feasible solution exists within the initial population). Classical penalty-based approaches were found not able to solve efficiently the constrained optimization problem here considered. Therefore, an advanced mechanism for handling infeasible solutions is required to mitigate the overall computational effort. In this study, the effectiveness of the archiving-based adaptive trade-off model (ArATM) [20] is investigated. Depending on the composition of $\mathcal{H}_{t}$, three situations can occur.

Feasible candidate solutions only. It might occur that $\mathcal{H}_{t}$ only includes feasible candidate solutions (e.g. at the end of the evolutionary search). In this case, feasible candidate solutions in $\mathcal{H}_{t}$ are sorted with reference to their objective function value, and the best $\mu$ individuals are selected for the parent population $\mathcal{P}_{t+1}$.

Infeasible candidate solutions only. When the feasible domain $\mathcal{D}$ is very small with respect to the search space $\mathcal{S}$, it might be possible that $\mathcal{H}_{t}$ includes infeasible candidate solutions only. In this case, the ArATM considers the objective function $f(\mathbf{x})$ and the degree of constraint violation $G(\mathbf{x})$ as two objectives which are examined simultaneously, thus obtaining a bi-objective optimization problem. The pseudo-code of the ArATM when infeasible individuals only occur in $\mathcal{H}_{t}$ is given in Algorithm 3 (the symbol $|\cdot|$ is the cardinality of the set between the vertical bars).

Both infeasible and feasible individuals. The procedure for this case is the following. First, the indices of the feasible individuals and those of the infeasible candidate solutions are stored into two sets, namely $\mathcal{Z}_{1}$ and $\mathcal{Z}_{2}$. Moreover, the best feasible individual $\mathbf{x}_{b e s t}$ and the worst feasible individual $\mathbf{x}_{\text {worst }}$ are identified. Subsequently, the normalized objective function $\bar{f}\left(\mathbf{x}_{i}\right)$ is calculated as follows:

$$
\bar{f}\left(\mathbf{x}_{i}\right)=\frac{f^{\prime}-f_{\min }^{\prime}}{f_{\max }^{\prime}-f_{\min }^{\prime}}
$$




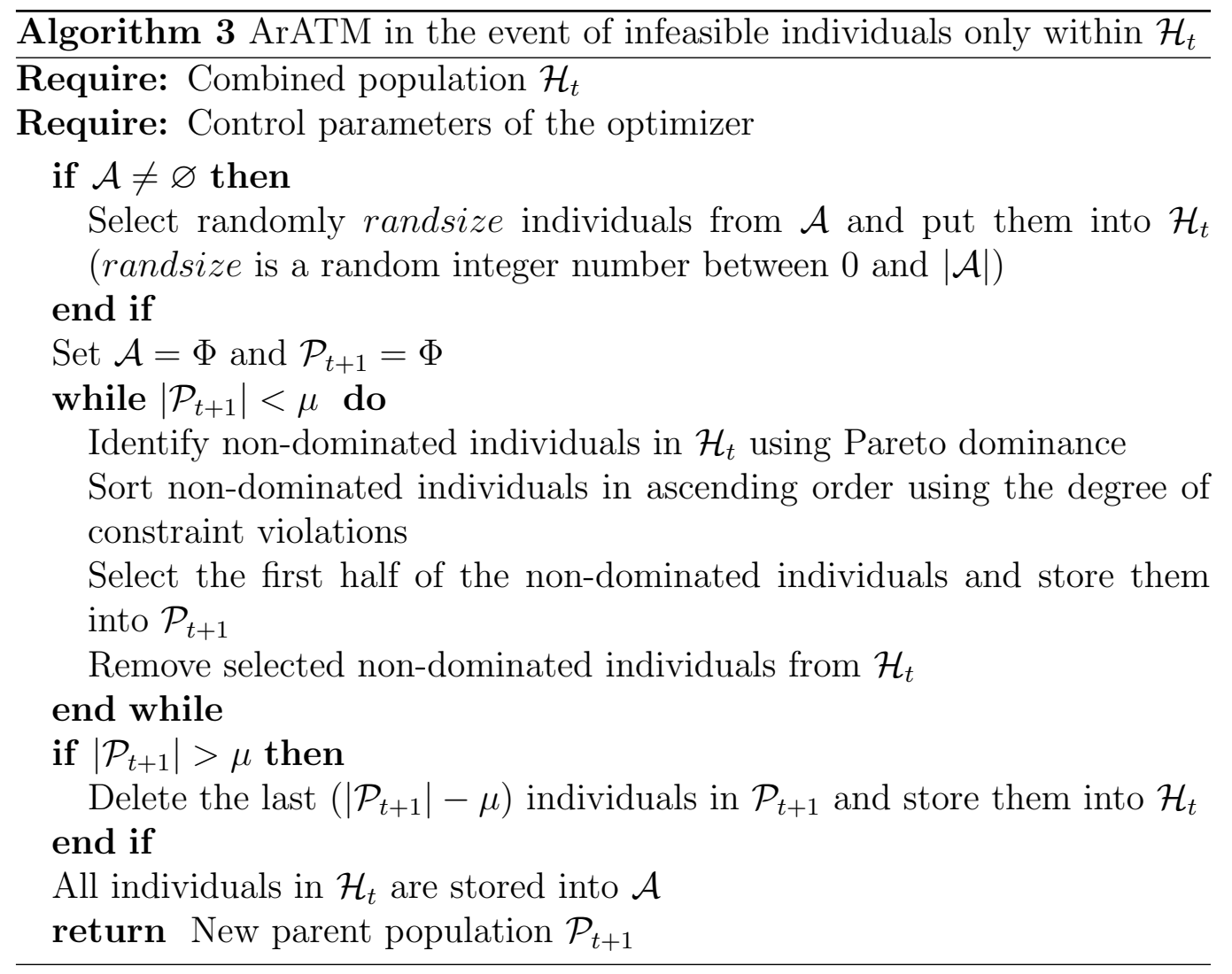


where

$$
\begin{gathered}
f^{\prime}= \begin{cases}f\left(\mathbf{x}_{i}\right) & \text { if } i \in \mathcal{Z}_{1} \\
\max \left\{\varphi f\left(\mathbf{x}_{\text {best }}\right)+(1-\varphi) f\left(\mathbf{x}_{\text {worst }}\right), f\left(\mathbf{x}_{i}\right)\right\} & \text { if } i \in \mathcal{Z}_{2}\end{cases} \\
f_{\text {max }}^{\prime}=\max _{z \in \mathcal{Z}_{1} \bigcup \mathcal{Z}_{2}}\left\{f^{\prime}\left(\mathbf{x}_{z}\right)\right\} \\
f_{\text {min }}^{\prime}=\min _{z \in \mathcal{Z}_{1} \bigcup \mathcal{Z}_{2}}\left\{f^{\prime}\left(\mathbf{x}_{z}\right)\right\} .
\end{gathered}
$$

The symbol $\varphi$ in Eq. (20a) is the feasibility proportion of the combined population $\mathcal{H}_{t}$. The normalized degree of constraint violation of each candidate solution is defined with reference to the flag variable criterion, thus obtaining:

$$
\bar{G}= \begin{cases}0 & \text { if } i \in \mathcal{Z}_{1} \\ \frac{G\left(\mathbf{x}_{i}\right)-G_{\min }}{G_{\max }-G_{\min }} & \text { if } i \in \mathcal{Z}_{2} \text { and criterion }=1 \\ G\left(\mathbf{x}_{i}\right) & \text { if } i \in \mathcal{Z}_{2} \text { and criterion }=2\end{cases}
$$

where

$$
\begin{aligned}
G_{\text {max }} & =\max _{z \in \mathcal{Z}_{2}}\left\{G\left(\mathbf{x}_{z}\right)\right\} \\
G_{\text {min }} & =\min _{z \in \mathcal{Z}_{2}}\left\{G\left(\mathbf{x}_{z}\right)\right\} .
\end{aligned}
$$

It is understood that $G\left(\mathbf{x}_{i}\right)$ is evaluated as in Eq. (14) if criterion $=1$ or as in Eq. (15) if criterion $=2$. The final fitness function $f_{\text {final }}\left(\mathbf{x}_{i}\right)$ is then calculated $\forall i$ as follows:

$$
f_{\text {final }}\left(\mathbf{x}_{i}\right)=\bar{f}\left(\mathbf{x}_{i}\right)+\bar{G}\left(\mathbf{x}_{i}\right)
$$

and $\mu$ individuals with the smallest $f_{\text {final }}\left(\mathbf{x}_{i}\right)$ value are selected to create the next parent population $\mathcal{P}_{t+1}$.

\section{Numerical application}

The optimization of the force-limiting floor anchorage system for a prototype RC dual wall-frame building is presented in the following. Final results are discussed to evaluate the effectiveness of the seismic protection strategy in comparison with the performance of the same structural system but with traditional cast-in-situ rigid connections between LFRS and GLRS. 


\subsection{Prototype structure}

The prototype RC structure is a 12-storey residential building with a footprint of $55 \mathrm{~m} \times 30 \mathrm{~m}$. The LFRS consists of two exterior shear walls along the longitudinal direction and two interior shear walls along the transversal direction. The inter-storey height is equal to $4.88 \mathrm{~m}$ for the ground floor whereas it is equal to $3.20 \mathrm{~m}$ for all remaining floors. Columns' section is $0.76 \mathrm{~m} \times 0.76 \mathrm{~m}$ from ground floor to 6th floor, and it is equal to $0.56 \mathrm{~m}$ $\times 0.56 \mathrm{~m}$ from 6 th to 12 th floor. The transversal section of the shear wall is $13.40 \mathrm{~m} \times 0.50 \mathrm{~m}$ and the $\mathrm{RC}$ slab has a thickness of $0.20 \mathrm{~m}$. Structural regularity in plan is guaranteed by the symmetry with respect to the two horizontal orthogonal axes. All the vertical elements are continuous from the foundation to the top of the building. Due to a higher ground storey height with respect to the floors above, the lateral stiffness of this floor is about $30 \%$ of the stiffness of the first floor. The geometry of the RC building is shown in Figure 2.

\subsection{Structural model and analysis}

An equivalent bidimensional finite element model was developed considering frames and shear walls acting along the transverse direction of the building. All structural members are modeled as elastic beam elements with an equivalent gross section defined as follows:

- 6 column sections for the equivalent exterior and interior gravity columns (GLRS),

- 5 diaphragm strip sections for the equivalent beams (GLRS), and

- 2 shear wall sections for the equivalent shear wall (LFRS).

Cracked sections are considered. Hence, the inertia of the cracked sections $I_{c r}$ is obtained by reducing the inertia of the uncracked section $I_{g}$ by $30 \%$ for the gravity columns, $75 \%$ for the beams and $65 \%$ for the shear wall. The equivalent bidimensional finite element model model is depicted in Figure 3. By assuming a rigid connection between LFRS and each floor diaphragm, the first three eigenperiods are $1.374 \mathrm{~s}, 0.229 \mathrm{~s}$ and $0.105 \mathrm{~s}$.

The OpenSees platform has been used to assess the response of the structural system by performing nonlinear dynamic analyses on a preselected set of spectrum compatible accelerograms. Rayleigh damping is assumed with a critical damping ratio equal to $2 \%$ considering first and third mode. The 
Building floor

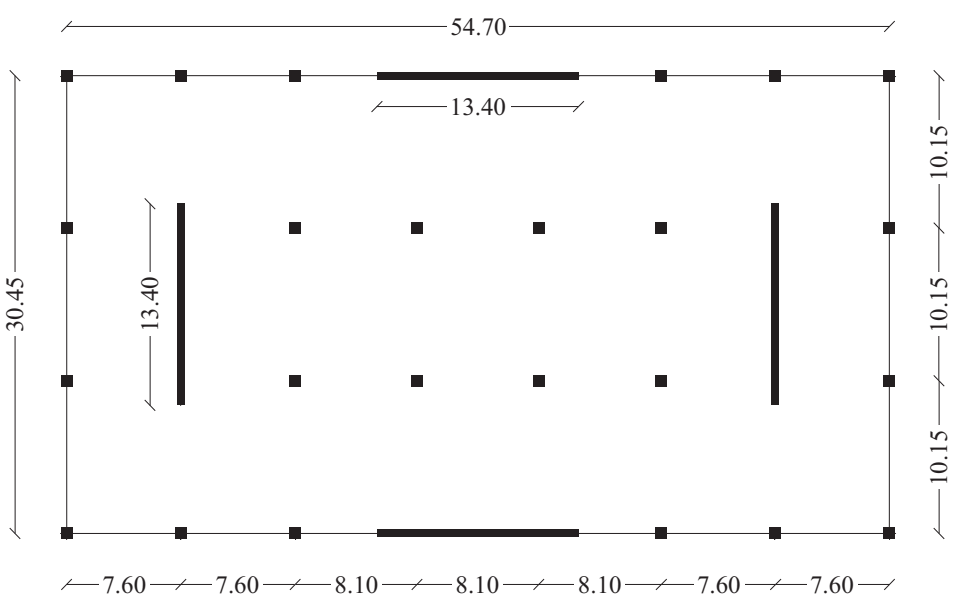

Shear wall

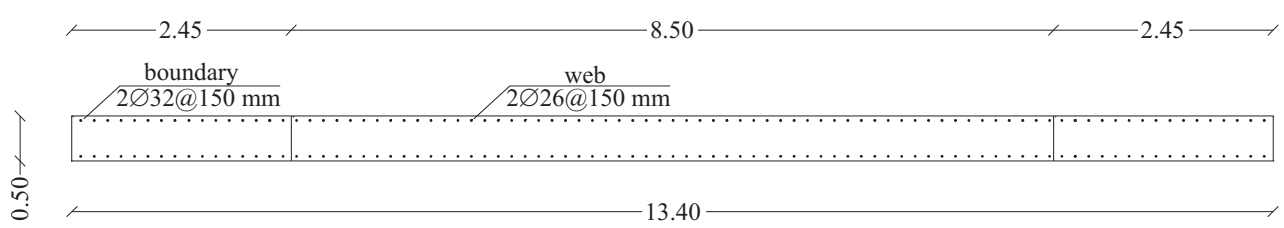

Figure 2: Geometry of the prototype RC building (geometrical measures of the building in meters, dimensions related to the reinforcement in millimeters). 


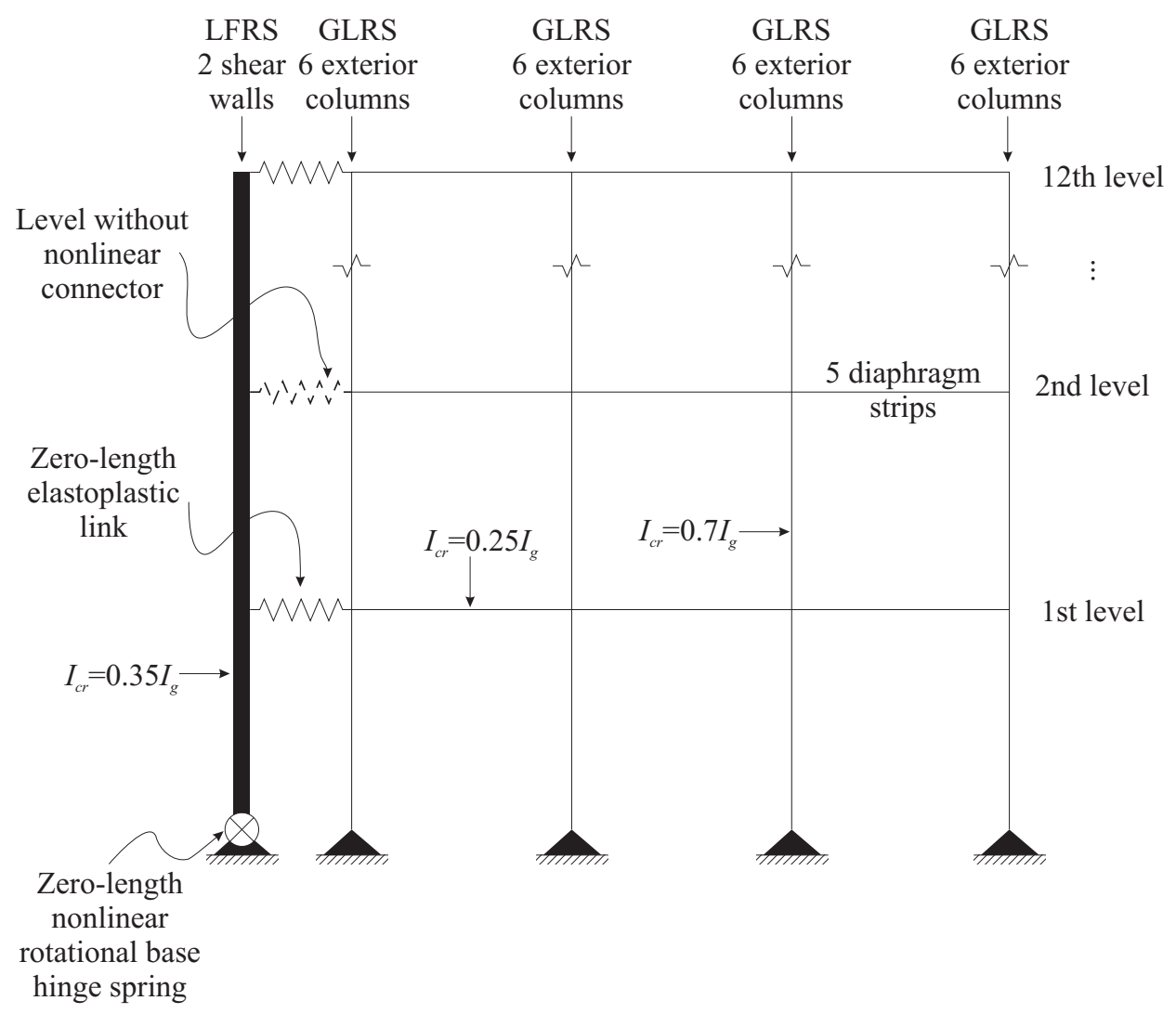

Figure 3: The equivalent 2D model of the prototype $\mathrm{RC}$ building. 
gravity load is applied (dead loads $+25 \%$ live loads) in order to take into account the second order effects (P-Delta effects). The nonlinear behavior of the structural system is allowed and concentrated at the connection between LFRS and GLRS as well as at the base of the shear wall. Zero-length nonlinear elements with an elastic-perfectly plastic behavior (uniaxialMaterial Hardening from OpenSees) are selected to model the floor connectors, while the base of the shear walls is modeled as a zero-length rotational hinge with pinching-type behavior (uniaxialMaterial Pinching4 from OpenSees). In particular, the parameters of the hysteretic law representing the shear wall response were calibrated so as to match the results from a cyclic test performed on precast shear walls in [24]. The initial stiffness of the floor connectors in $[\mathrm{kN} / \mathrm{m}]$ is the design variable of the optimization procedure whereas it is assumed that the yield strength is a linear function of the initial stiffness. Specifically, the yield strength value $F_{y}$ is obtained by dividing the initial stiffness by a predefined constant factor.

Natural accelerograms were selected within the seismic inputs available at the PEER NGA database. They have been scaled so as to match the design response spectrum having a peak ground acceleration (PGA) equal to 0.50 g. In this regard, the technique selected for this study is the linear scaling of the seismic inputs. The median spectrum calculated from all the spectra of the selected accelerograms has to satisfactory match the design response spectrum within the relevant range of periods of the structural system with rigid connections (i.e., 0.6-2.0 s). Six natural earthquake records have been considered for the present numerical optimization. They are representative of earthquakes recorded at different locations, i.e. Kobe (EQ1), Loma Prieta (EQ2, EQ3) and Northridge (EQ4, EQ5, EQ6). The corresponding spectra are showed in Figure 4.

The $(\mu+\lambda)$-constrained differential evolution algorithm has been implemented in MATLAB, and suitable scripts were developed to allow the interaction with OpenSees. For this case-study, the optimization problem consists of 48 constraints. A damage limitation limit state is considered, for which a seismic action having a larger probability of occurrence than the design seismic action associated with the ultimate limit state has to be assumed. For this reason, a reduction factor for the accelerograms equal to 0.5 is selected in order to take into account the lower return period of the earthquake combined with the damage limitation requirements. The maximum allowable drift for, both, LFRS and GLRS is $0.5 \%$ of the inter-storey height. The maximum acceleration of the GLRS at the floors level is $0.20 \mathrm{~g}$ whereas the 


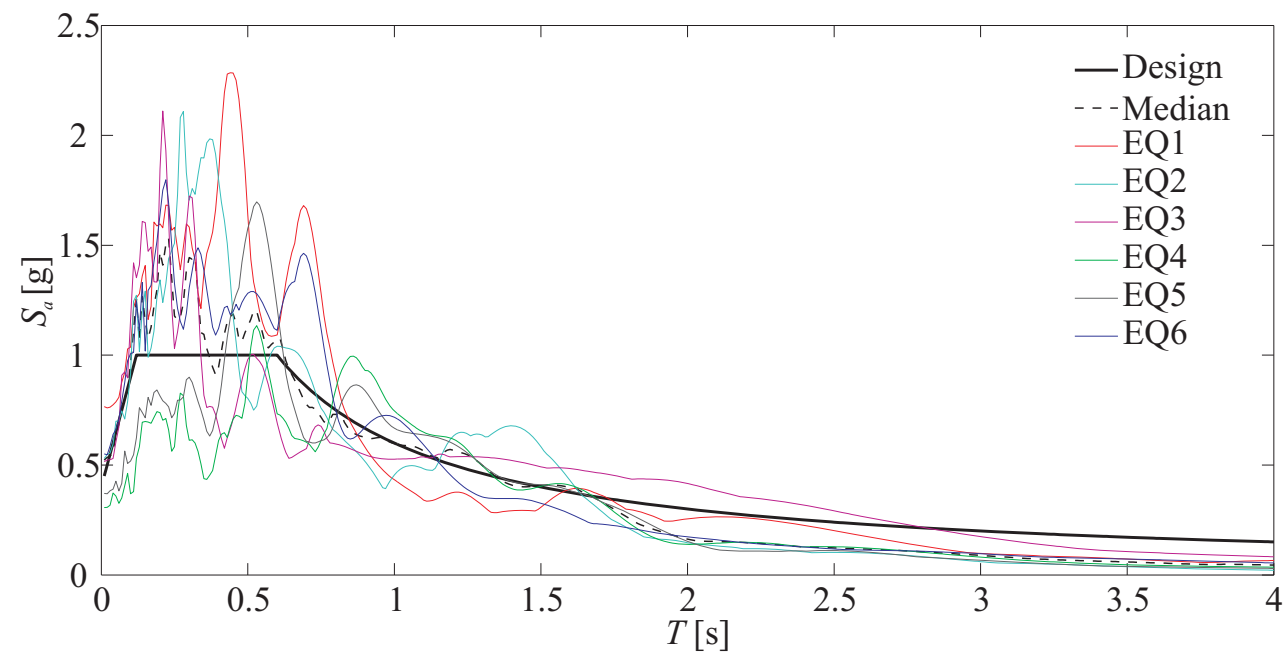

Figure 4: Spectra of the considered ground acceleration time histories, together with the median value and the design spectrum.

sliding limit is $0.25 \mathrm{~m}$. Threshold values for interstorey drift and floor acceleration approximately correspond to light structural damage scenario in moment resisting frames and their contents [25]. The total number of design variables is 12 , and a wide search space has been considered. The maximum and the minimum values of the link stiffness are $5,000 \mathrm{kN} / \mathrm{m}$ and 500,000 $\mathrm{kN} / \mathrm{m}$, respectively. Control parameters of the optimizer are listed in Tab. 1. The total number of fitness evaluations is 5,075 .

\subsection{Results}

The final optimal solution $\mathbf{x}^{*}$ after 5,075 fitness evaluations includes the following no-null elements: $x_{1}^{*}=171,461.55 \mathrm{kN} / \mathrm{m}\left(F_{y, 1}^{*}=198.45 \mathrm{kN}\right), x_{3}^{*}=$ $416,942.49 \mathrm{kN} / \mathrm{m}\left(F_{y, 3}^{*}=482.57 \mathrm{kN}\right), x_{5}^{*}=179,080.99 \mathrm{kN} / \mathrm{m}\left(F_{y, 5}^{*}=207.27\right.$ $\mathrm{kN}), x_{11}^{*}=189,604.48 \mathrm{kN} / \mathrm{m}\left(F_{y, 11}^{*}=219.45 \mathrm{kN}\right)$. This means that the optimal configuration of the protection system is based on the installation of connectors at the first, third, fifth and eleventh floor (i.e., 1/3 of the total number of floors has to be equipped with floor connectors). Therefore, there is no need to equip every single floor with such elasto-plastic devices, as it was also observed in the optimum design of links between adjacent buildings [16]. Convergence curves of either feasibility proportion and objective function are shown in Figure 5. The trend of the feasibility proportion demonstrates the complexity of the constrained optimization problem, since no feasible solution 


\begin{tabular}{ll}
\hline Parameter & Value \\
\hline$\mu$ & 35 \\
$\lambda$ & 105 \\
$F$ & 0.80 \\
$C R$ & 0.90 \\
$P$ & 0.50 \\
$P M$ & 0.05 \\
$\eta$ & 0.05 \\
$\kappa$ & 0.60 \\
\hline
\end{tabular}

Table 1: Control parameter values for $(\mu+\lambda)$-constrained differential evolution algorithm.

is within the initial population. Nonetheless, the search strategy has been able to move all candidate solutions within the optimal feasible region after 1,000 fitness evaluations. The final objective function value is about $40 \%$ of the initial one (the objective function value of the solution with the smallest degree of constraints violation), highlighting a significant cost reduction with respect to best initial design.

The performance of the building proctected by means of the optimal forcelimiting floor anchorage system is shown in the following Figure 6 (earthquake record EQ1), Figure 7 (earthquake record EQ2), Figure 8 (earthquake record EQ3), Figure 9 (earthquake record EQ4), Figure 10 (earthquake record EQ5) and Figure 11 (earthquake record EQ6). Results carried out with the optimal structural configuration ("Optimum") are compared with the performance of the building provided with rigid links at each storey ("Rigid"). To better understand the working principle of the considered seismic protection strategy, it is also useful to include the results corresponding to a very flexible link between GLRS and LFRS. To this end, the behavior corresponding to the minimum links configuration needed for the convergence of the nonlinear dynamic analysis ("Minimum") is considered (this consists of 2 link devices with minimum stiffness value placed at the 4 th and 9 th floor). Finally, "Threshold" indicates the maximum admissible value of the corresponding constraint.

This comparative analysis demonstrates that the non-optimal configurations are not able to satisfy the selected limit states for all the earthquake records and, according to [25], they might lead the building to experience moderate or heavy damages. Overall, optimally designed nonlinear floor 

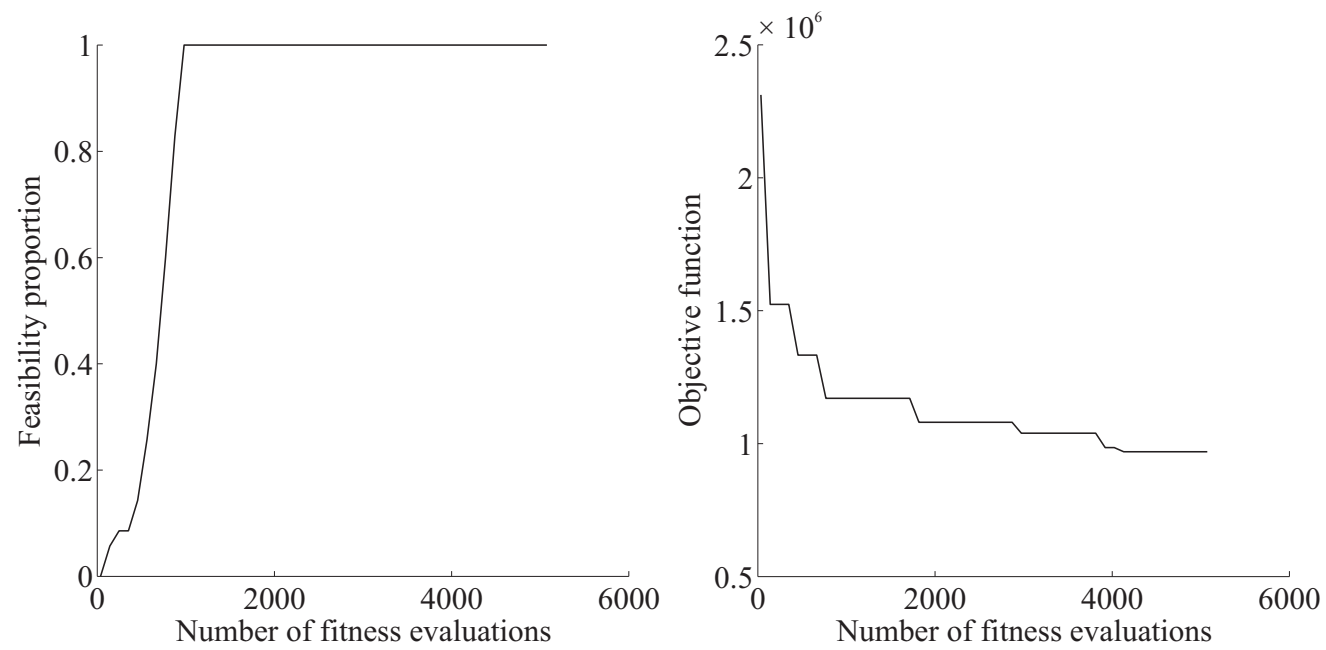

Figure 5: Convergence of feasibility proportion (left) and objective function (right).
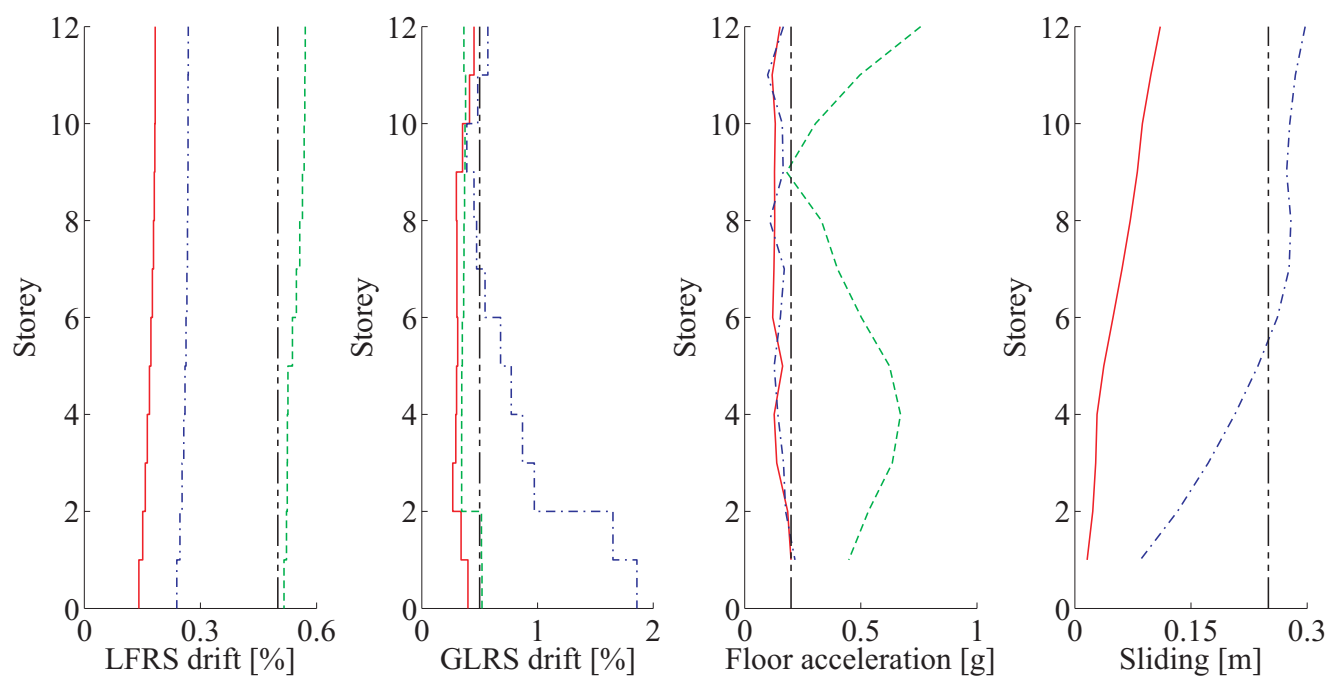

- Optimum

-....-Minimum

------- Rigid _--- - Threshold

Figure 6: Structural performance for the earthquake record EQ1. 

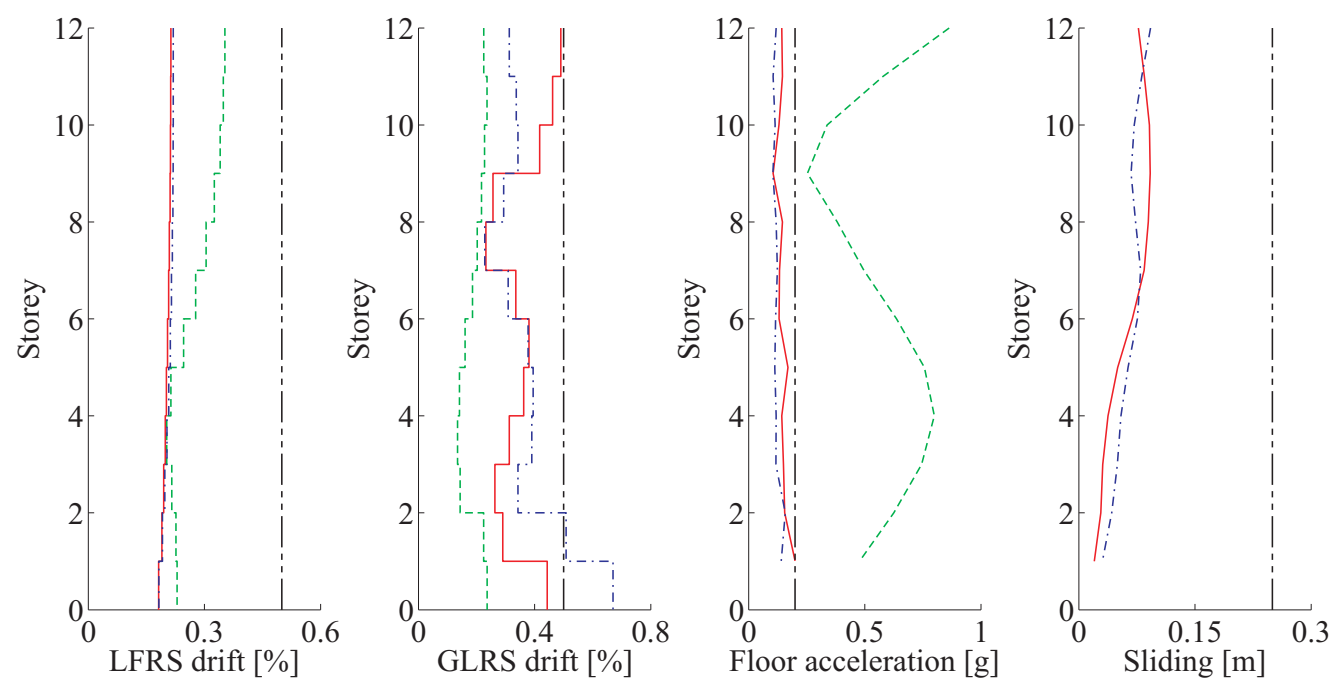

Optimum

Minimum

- Rigid _-.- Threshold

Figure 7: Structural performance for the earthquake record EQ2.
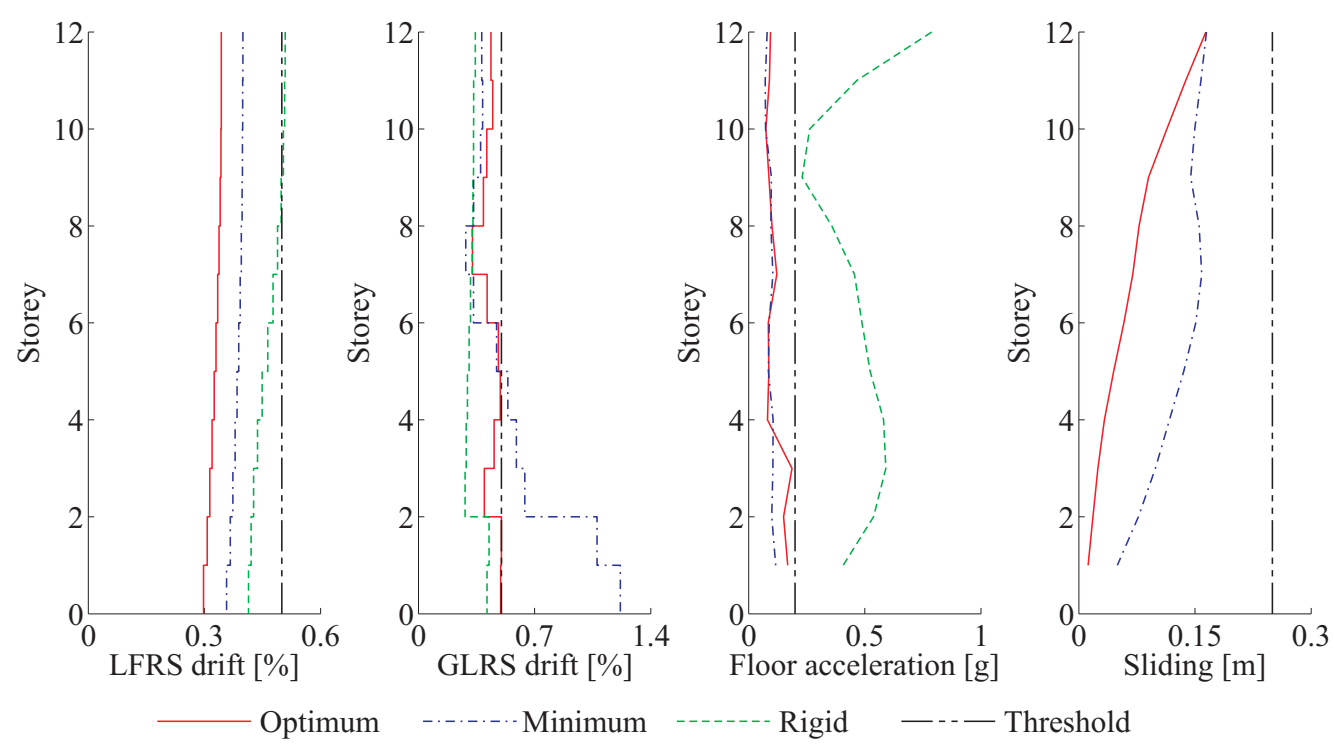

Figure 8: Structural performance for the earthquake record EQ3. 

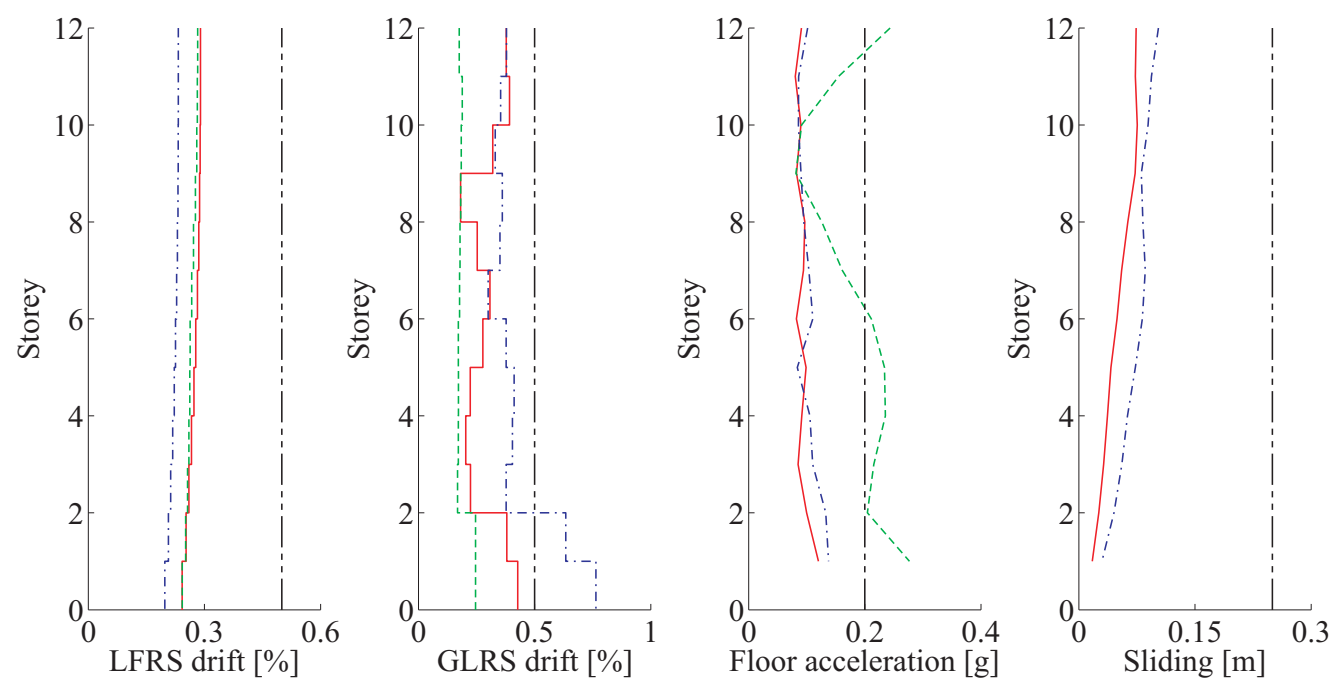

Rigid _-.- Threshold

Figure 9: Structural performance for the earthquake record EQ4.
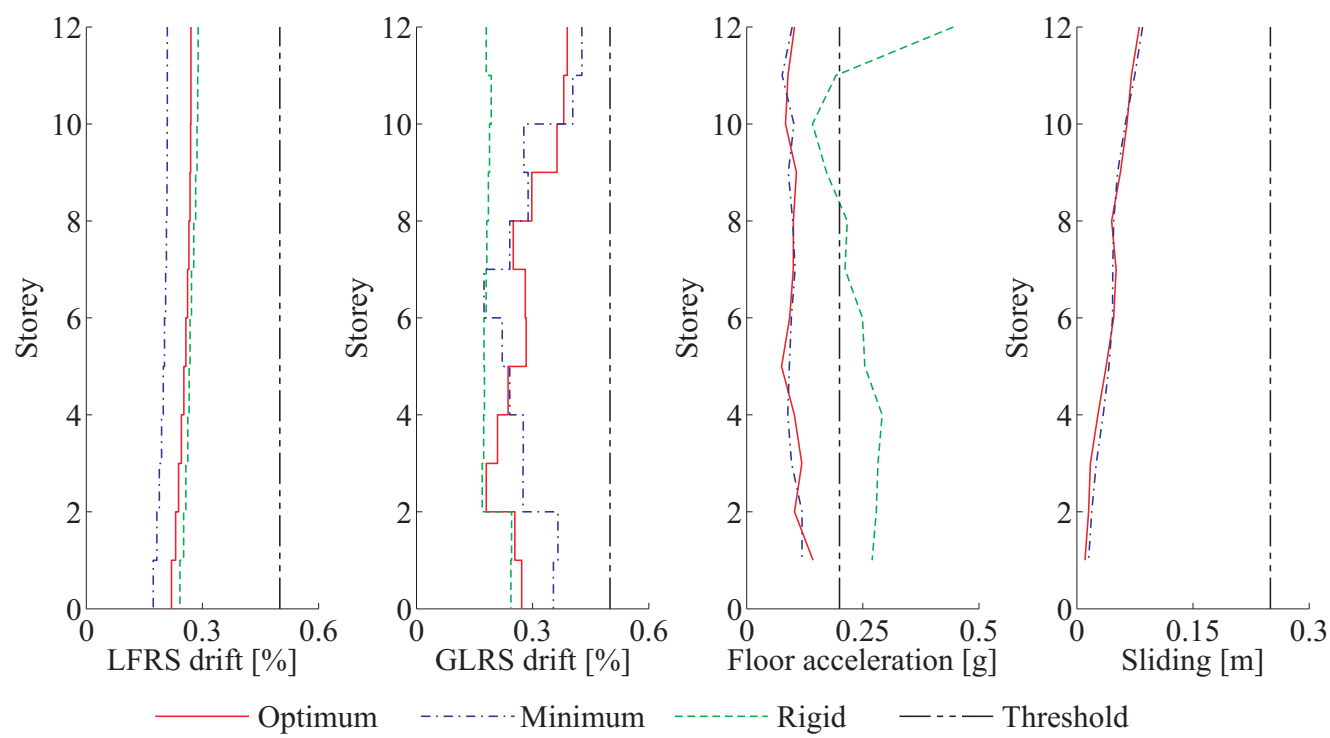

Figure 10: Structural performance for the earthquake record EQ5. 

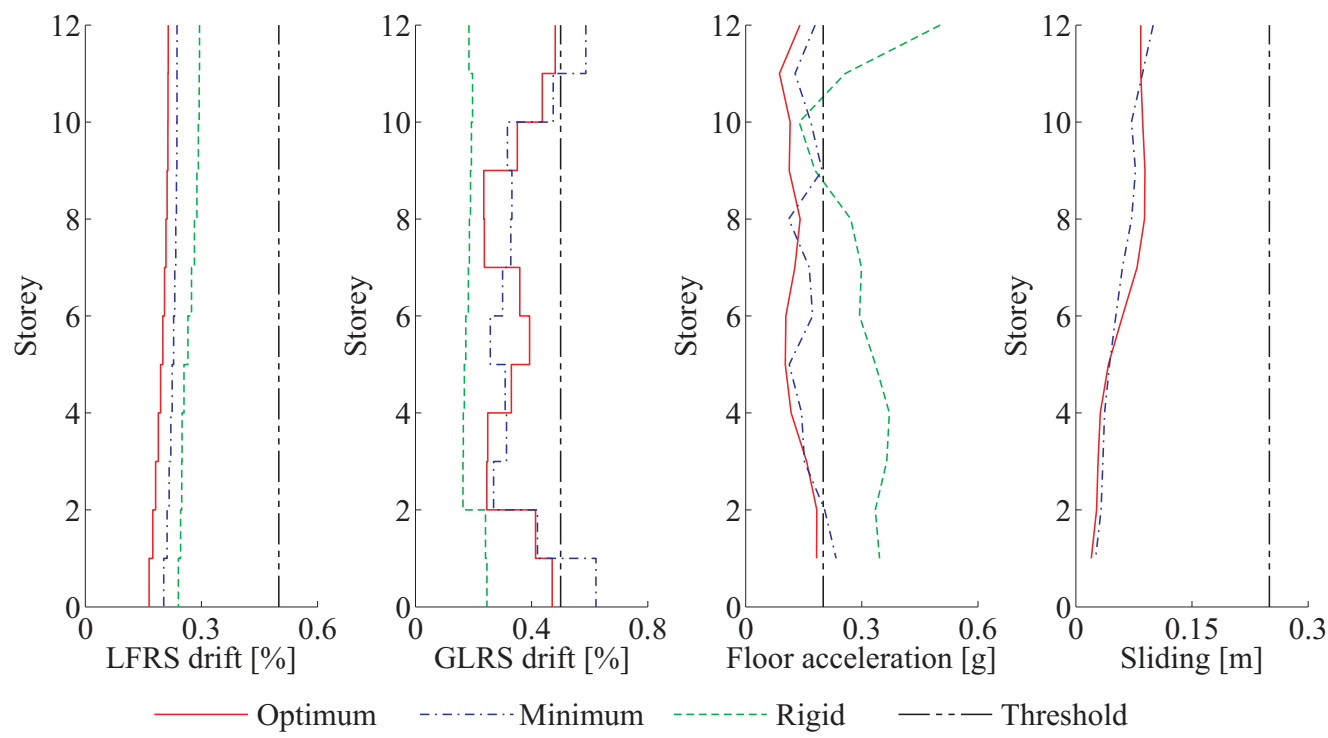

Figure 11: Structural performance for the earthquake record EQ6.

connectors lead to performances intermediate between those corresponding to traditional cast-in-situ rigid connections and those obtained by assuming a very flexible link. As expected, the presence of a rigid connection between the GLRS and the LFRS has a negative influence on the floor acceleration demand values, with evident amplification effects. The placement of nonlinear floor connectors between GLRS and LFRS has great impact in reducing the floor accelerations, and consequently in reducing the severity of the damage to the floor system and its contents. The LFRS drift is occasionally reduced by placing such nonlinear floor connectors. This damage mitigation for the LFRS is clearly beneficial, especially because it helps the reduction of the accumulated structural damage in case of aftershocks or further seismic events during the design lifetime of the building, enhancing in this way the global structural safety. A more economical structural repair will be eventually required for the shear wall (LFRS) because of the damage reduction. On the contrary, the use of optimized flexible devices between GLRS and LFRS causes an increment of the GLRS drift values with respect to the system with rigid connections, since the gravity frame is no more rigidly linked to the stiffer shear wall that acts as a lateral deformation constraint for the GLRS. As a consequence, reinforcement details for the GLRS have to be properly designed in order to account for the slight increment in the 
inter-storey drift demand. Considering the class of buildings to which this seismic protection strategy is intended and taking into account the increment of the costs attributable to the installation of the nonlinear connectors, the expenses to accommodate higher ductility demands at the columns' sections of the ground storey (i.e., by providing more confinement through transversal reinforcement at a smaller spacing) will be rather modest. The increment of the GLRS drift value is especially relevant for the ground floor because of its higher storey height. A certain displacement ductility is therein required in order to avoid failure of the base columns or any soft-storey mechanism. In this sense, it is not surprising that the optimal solution includes one link at the first slab. By virtue of the constrained optimization procedure, however, the GLRS drift values lie below the given threshold. Conversely, a too flexible or inexistent connection between GLRS and LFRS causes inter-storey drift values of the GLRS larger than the selected threshold, even if it results beneficial in reducing the floor accelerations.

It is evident that the optimal configuration fulfills all problems' constraints for all earthquake records and it can be considered as a compromise among these limit configurations. The optimized distribution of floor connectors is able - acting together with the shear walls (LFRS) - to limit the inter-storey drift of the GLRS (especially at the ground floor), whereas the relative displacement between GLRS and LFRS is capable to mitigate the floor accelerations of the GLRS and the structural damages of the LFRS.

As the results clearly shown, the optimization procedure is mostly influenced by the inter-storey drift of the GLRS. This was actually expected considering the fact that the inter-storey height of the ground floor $(4.88 \mathrm{~m})$ is sensibly higher than the inter-storey height of the floors above $(3.20 \mathrm{~m})$. This non uniform distribution of the stiffness between the columns at the base and the columns in the upper floors causes a soft storey-type behavior for the GLRS, i.e. higher inter-storey drift, chord rotation and transverse ductility demands at the base with a consequent concentration of the damage at the ground floor.

Finally, the presence of relative displacement between LFRS and GLRS brings the attention on the design of the floor connectors that are required to provide the optimal strength and stiffness. They are also required to remain stable (no softening behavior) before reaching their predefined maximum displacement capacity. 


\section{Conclusions}

This paper has addressed the optimum design of a new strategy for mitigating the detrimental effects of seismic loads on dual wall-frame buildings. The considered seismic protection system is intended to decouple the dynamic response of the lateral force resisting system (LFRS) from that of the gravity load resisting system (GLRS). The relative displacement under seismic action between LFRS and GLRS allows the dissipation of energy through elasto-plastic floor connectors. The design of such passive protection system is posed as constrained optimization problem where the objective function to be minimized is related to the total manufacturing cost of the devices. Constraints deal with the performance of the entire structural system, whereas selected design variables are the link positions and their mechanical properties. Final results for a prototype 12-storey dual wall-frame reinforced concrete building have demonstrated its efficacy in reducing the damage due to seismic loading. Specifically, the use of nonlinear connectors between LFRS and GLRS has positive influence in reducing the damage severity of the floor system and building contents due to excessive acceleration demand values. Occasionally, it has been shown that this protection strategy is also able to mitigate the structural damage of the LFRS by reducing its drift values. An increment of the GLRS drift values is also observed with respect to traditional cast-in-situ rigid connections between LFRS and GLRS. This negative effect has to be taken into account by enhancing the ductility of the GLRS through proper reinforcement details. Indeed, the definition of GLRS is no longer literally appropriate since a certain amount of the horizontal forces is transmitted from the LFRS to the GLRS through the floor connectors. On the other hand, the most of the shear capacity still relies on the larger stiffness of the shear wall. Therefore, the conceptual separation into LFRS and GLRS seems still acceptable. A consequence of the lateral drift experienced by the GLRS is the need of considering the P-Delta effects for the ground storey columns. At the foundation level, an increment of the drift demand for the ground storey, together with the presence of gravity loads, will cause an increment of the flexural demand.

An important issue for this work is the numerical resolution of the optimization problem. It is worth noting that most researches adopt simplified assumptions in the optimum design of seismic protection systems, e.g. linear (or linearized) shear-type systems under random vibrations. This kind of simplification has not been assumed here, and the final constrained optimum 
design of the force-limiting floor anchorage system is supported by nonlinear dynamic analyses. An analytical closed form design solution is no longer possible under such assumptions. Therefore, a numerical optimization procedure has been implemented in MATLAB whereas the OpenSees platform has been used to perform nonlinear dynamic analyses. On rejecting the hypothesis of linear behavior, the final computational time grows drastically. However, the adopted optimization procedure based on the $(\mu+\lambda)$-constrained differential evolution algorithm has proved to be efficient.

\section{Acknowledgments}

The presented work is part of the National Science Foundation (NSF) George E. Brown Network for Earthquake Engineering Simulation Research (NEESR) project "Inertial Force-Limiting Floor Anchorage Systems for Seismic Resistant Building Structures" being led at the University of Arizona by Principal Investigator Prof. Robert B. Fleischman, with co-investigators Prof. Jose I. Restrepo at University of California San Diego and Prof. Richard Sause at Lehigh University. Any opinions, findings, conclusions, and recommendations expressed in this paper are those of the authors and do not necessarily reflect the views of the sponsor.

\section{References}

[1] D. K. Liu, Y. L. Yang, and Q. S. Li. Optimum positioning of actuators in tall buildings using genetic algorithm. Computers 85 Structures, 81(32):2823-2827, 2003.

[2] G. C. Marano and G. Quaranta. Robust optimum criteria for tuned mass dampers in fuzzy environments. Applied Soft Computing, 9(4):1232 $-1243,2009$.

[3] A. Y. T. Leung, H. Zhang, C. C. Cheng, and Y. Y. Lee. Particle swarm optimization of TMD by non-stationary base excitation during earthquake. Earthquake Engineering and Structural Dynamics, 37(9):12231246, 2008.

[4] F. A. C. Viana, G. I. Kotinda, D. A. Rade, and V. Steffen Jr. Tuning dynamic vibration absorbers by using ant colony optimization. Computers \& Structures, 86(13-14):1539-1549, 2008. 
[5] G. Falsone and G. Ferro. Best performing parameters of linear and nonlinear seismic base-isolator systems obtained by the power flow analysis. Computers \& Structures, 84(31-32):2291 - 2305, 2006.

[6] G. Quaranta, G. C. Marano, R. Greco, and G. Monti. Parametric identification of seismic isolators using differential evolution and particle swarm optimization. Applied Soft Computing, 22:458 - 464, 2014.

[7] J. E. Luco and F. C. P. De Barros. Optimal damping between two adjacent elastic structures. Earthquake Engineering 85 Structural Dynamics, 27(7):649-659, 1998.

[8] Y. L. Xu, Q. He, and J. M. Ko. Dynamic response of damper-connected adjacent buildings under earthquake excitation. Engineering Structures, 21(2):135 - 148, 1999.

[9] H. P. Zhu and Y. L. Xu. Optimum parameters of Maxwell model-defined dampers used to link adjacent structures. Journal of Sound and Vibration, 279(12):253 - 274, 2005.

[10] A.V. Bhaskararao and R.S. Jangid. Seismic response of adjacent buildings connected with friction dampers. Bulletin of Earthquake Engineering, 4(1):43-64, 2006.

[11] Y. Q. Ni, J. M. Ko, and Z. G. Ying. Random seismic analysis of adjacent buildings coupled with non-linear hysteretic dampers. Journal of Sound and Vibration, 246(3):403 - 417, 2001.

[12] M. Basili and M. De Angelis. A reduced order model for optimal design of 2-mdof adjacent structures connected by hysteretic dampers. Journal of Sound and Vibration, 306(12):297 - 317, 2007.

[13] M. Basili and M. De Angelis. Optimal passive control of adjacent structures interconnected with nonlinear hysteretic devices. Journal of Sound and Vibration, 301(12):106 - 125, 2007.

[14] C. P. Pantelides and F. Y. Cheng. Optimal placement of controllers for seismic structures. Engineering Structures, 12(4):254 - 262, 1990.

[15] S.-Y. Ok, J. Song, and K.-S. Park. Optimal design of hysteretic dampers connecting adjacent structures using multi-objective genetic algorithm 
and stochastic linearization method. Engineering Structures, 30(5):1240 - 1249, 2008.

[16] M. E. Uz and M. N. S. Hadi. Optimal design of semi active control for adjacent buildings connected by MR damper based on integrated fuzzy logic and multi-objective genetic algorithm. Engineering Structures, 69:135 - 148, 2014.

[17] K. Deb and S. Gulati. Design of truss-structures for minimum weight using genetic algorithms. Finite Elements in Analysis and Design, 37(5):447-465, 2001.

[18] D. C. Charmpis, P. Komodromos, and M. C. Phocas. Optimized earthquake response of multi-storey buildings with seismic isolation at various elevations. Earthquake Engineering and Structural Dynamics, 41(15):2289-2310, 2012.

[19] S. Ganzerli, C. P. Pantelides, and L. D. Reaveley. Performance-based design using structural optimization. Earthquake Engineering 83 Structural Dynamics, 29(11):1677-1690.

[20] G. Jia, Y. Wang, Z. Cai, and Y. Jin. An improved $(\mu+\lambda)$-constrained differential evolution for constrained optimization. Information Sciences, 222:302-322, 2013.

[21] G. Quaranta, A. Fiore, and G. C. Marano. Optimum design of prestressed concrete beams using constrained differential evolution algorithm. Structural and Multidisciplinary Optimization, 49(3):441-453, 2014 .

[22] G. Quaranta, G. Monti, and G. C. Marano. Parameters identification of Van der Pol - Duffing oscillators via particle swarm optimization and differential evolution. Mechanical Systems and Signal Processing, 24(7):2076-2095, 2010.

[23] S. Guo and C. Yang. Enhancing differential evolution utilizing eigenvector-based crossover operator. IEEE Transactions on Evolutionary Computation, 19(1):31-49, 2015. 
[24] T. Holden, J. Restrepo, and J. Mander. Seismic performance of precast reinforced and prestressed concrete walls. Journal of Structural Engineering, 129(3):286-296, 2003.

[25] C. C. Mitropoulou, N. D. Lagaros, and M. Papadrakakis. Building design based on energy dissipation: a critical assessment. Bulleting of Earthquake Engineering, 8(6):1375-1396, 2010. 


\section{List of Figures}

1 Placement of links between the LFRS and the GLRS: adopted strategy to identify operative floor connectors within a bidimensional search space. . . . . . . . . . . . . . . . . . 6

2 Geometry of the prototype RC building (geometrical measures of the building in meters, dimensions related to the reinforcement in millimeters). . . . . . . . . . . . . . . . . . 18

3 The equivalent 2D model of the prototype RC building. . . . . 19

4 Spectra of the considered ground acceleration time histories, together with the median value and the design spectrum. . . . 21

5 Convergence of feasibility proportion (left) and objective function (right). . . . . . . . . . . . . . . . . . 23

$6 \quad$ Structural performance for the earthquake record EQ1. . . . . 23

$7 \quad$ Structural performance for the earthquake record EQ2. . . . . 24

8 Structural performance for the earthquake record EQ3. . . . . 24

$9 \quad$ Structural performance for the earthquake record EQ4. . . . . 25

10 Structural performance for the earthquake record EQ5 . . . . . 25

11 Structural performance for the earthquake record EQ6. . . . . 26

\section{List of Tables}

1 Control parameter values for $(\mu+\lambda)$-constrained differential evolution algorithm. . . . . . . . . . . . . . 22 